\title{
PARTICULARITIES IN THE CHILD CHRONIC RHINOSINUSITIS
}

\author{
Alina Murgu ${ }^{1}$, Evelina Moraru ${ }^{1}$, Daniela Rusu ${ }^{2}$, Elena Macsim $^{3}$, Ileana Ioniuc ${ }^{1}$, \\ Bogdan Stana ${ }^{1}$, Alice Azoicai ${ }^{1}$ \\ ${ }^{1}$ II-nd Pediatrics Clinic, "St. Maria” Emergency Hospital for Children, \\ "Gr. T. Popa" University of Medicine and Pharmacy, Iasi \\ ${ }^{2}$ ENT Department, "St. Maria” Emergency Hospital for Children, \\ "Gr. T. Popa" University of Medicine and Pharmacy, Iasi \\ ${ }^{3}$ Department of Radiology, "St. Maria” Emergency Hospital for Children, Iasi
}

\begin{abstract}
Chronic rhinosinusitis is a rare pathology in pediatric pathology versus adult patients. Clinical manifestations are related, on the one hand, with the anatomical particularities and, on the other hand, with the immune response correlated with the age of the child. Allergy is the main cause in $50 \%$ of child rhinitis, $40 \%$ of them debuting early until the age of 6 years. The clinical expression of allergic rhinosinusitis in children can sometimes be accompanied by comorbidity manifestations or complications. Impaired mucociliary clearance can be induced by other rare pathological situations that produce mucus rheology modification, as in cystic fibrosis (CF). Through, the clinical heterogenicity of expression is in relation, on the one hand, with immunogenic response and anatomical particularities relative to age of the child, and secondly with the diversity of inducing factors (from very frequent like allergy to the least frequent, CF). It requires a correct diagnosis, early and appropriate treatment by a multidisciplinary team collaboration.
\end{abstract}

Keywords: rhinosinusitis, child, cystic fibrosis, allergy

Chronic rhinosinusitis, defined as chronic inflammation of nasal mucosa and paranasal sinuses lasting more than 3 months is a rare pathology in pediatric pathology versus adult patients, but recognizes a variety of causes that can sometimes constitute diagnostic pitfalls. In the US, 30 million adults are diagnosed annually with rhinosinusitis, but only $15 \%$ develop chronic rhinosinusitis vs. $0.5-5 \%$ of children who are diagnosed with acute rhinosinusitis, and of these only a small part will develop chronic rhinosinusitis (1). The most commonly affected in small children and infants are the ethmoidal sinuses and maxillar sinuses, and after 6 years of age- sphenoidal and frontal sinuses. In fact, the clinical manifestations are related, on the one hand, with anatomical particularities and, on the other hand, the immune response correlated with the age of the child. Infants frequently express the aspect of persistent rhinorrhea or recurrent dis- ease after upper respiratory tract infections (most often otitis, nasopharyngitis, laryngitis), with or without purulent conjunctival secretion as an expression of ethmoidal injury. The clinical picture is slightly contoured in preschool children (3-6 years) with chronic nasal obstruction and persistent runny nose that draws attention, with or without tonsillar hypertrophy, lymph node swelling and/or serous otitis sometimes. At older ages, the patient may present somewhat more frequently nonspecific symptoms such as chronic frontal headache, sleep disorders (obstructive sleep apnea) associated with irritability and/or daytime sleepiness, hyposmia, chronic cough, breathing on the mouth, dry mouth sensation, dysphagia, nocturnal snoring and nasal secretion. The core of the pathogenic mechanism triggered by a variety of factors is essentially the altered mucociliary clearance by inflammation of the sinuses. Consequently, there will be blocking in

Corresponding author:

Bogdan Stana, "Gr. T. Popa" University of Medicine and Pharmacy, 16 Universitatii Street, lasi

E-mail: bogdan.stana@gmail.com 
sinus ostium and drainage alteration with secondary respiratory secretions and stasis. Injuried ciliary epithelium has the effect of inducing secretion retention, bacterial superinfection - an aspect that creates the conditions and persistence of inflammation, thereby closing a vicious circle.

Etiological palette involved in chronic rhinosinusitis of the child are from the most common (infection, allergy, gastro-oesophageal reflux), to the rarest, as well as anatomical defects (velo-pallatine cleft, deviated septum etc.) or systemic causes (cystic fibrosis, ciliary dyskinesia, immune deficiencies etc.). Allergy is the main cause in $50 \%$ of child rhinitis, $40 \%$ of them debuting early until the age of 6 years. The incidence of chronic rhinosinusitis appreciated in a group of 4,044 children aged 8-9 years consulted in ENT department of Children's Hospital Boston (USA) in 10 years was $53.8 \%$, of which $26.9 \%$ were associated allergic rhinitis (2). Allergic sinusitis is rarely described in children vs. adult, a small percentage $(5-10 \%)$ of children aged less than 5 years being diagnosed with fungal sensitization (Bipolaris, Drechstera, Helminthosporium, Fusarium, Aspergillus spp) (3). Consequently, the child frequently has the expression of allergic rhinosinusitis, more often than a simple sinusitis. The risk for atopy is appreciated up to $40 \%$ if one parent is allergic, and up to $80 \%$ if both parents are affected. Although so far no genetic variant showed a constant association with allergic rhinosinusitis, involving genetic polymorphism, however, they have been described in relation to some HLA genes triggering pollen allergy. Regarding the pathogenic activation of TH2 via immune signals with specific cytokines (IL-4, IL-5, I19, IL13), and the switch from Th1 to Th2 in certain immunogenic circumstances, allowed the identification of other trigger factors, in addition to those already known, as the allergy (allergens, drugs, foods) as well as viral or bacterial infection, exercise, endocrine factors (4). Therefore, the clinical expression of allergic rhinosinusitis in children (sneezing, rhinorrhea, itching and nasal obstruction) can sometimes be accompanied by comorbidities of allergic asthma (40-50\% of cases), allergic conjunctivitis, gastroesophageal reflux, atopic dermatitis or complications in upper respiratory tract situses (serous otitis media, tubal dysfunction, adenoid hypertrophy, chronic tonsillitis, laryngitis, nasal polyposis) (5).

The correct diagnosis of allergic rhinosinusitis suggested by eosinophils in the nasal secretion, and eosinophils/PMN percentage, supported by allergy tests (specific IgE, total IgE, skin prick test) in con- junction with the examination of ENT (rinomanometry) and imagistic investigations (radiography or, ideally, tomography of the sinuses). Usually, the nasal biopsy is not a routine procedure, but immunohistochemical determination of eosinophilic cationic protein along with neutrophilic elastase dosage may clarify the type of inflammation. On the other hand, histopathology associated with bacterial tests can objectify allergic fungal mucin $(\mathrm{Mu}-$ cor mycosis), useful for the diagnosis of allergic fungal sinusitis (6). Framing the correct diagnosis, taking into account ARIA guide for allergic rhinitis enables individualized treatment, that minimizes complications which may arise in the evolution of these patients-obstructive sleep apnea, chronic nasal obstruction, anosmia, headache, pharingeal chronic irrittation or severe risk evolutive complications, as a consequence of secondary bacterial infection - orbital cellulitis, cavernous sinus thrombosis, brain abscess, osteomyelitis, meningitis, sepsis. Allergic rhinosinusitis treatment is actually the same with allergic rhinitis and has its aims like the control of the symptoms, improving the child quality of life, without altering the functional skills, as well as preventing complications. The means to treat these patients are represented by associating optimal therapeutic efficacy with control over the patient's living environment, and pharmaceutical specific immunotherapy (modulating immune response through IL10 to IgG4 synthesis). Controller pharmacological treatment is based on topical corticosteroid nasal administratrion, with or without antihistaminic agents (nasal or oral), leukotriene modifiers and/or nasal chromone therapy (7).

However, alteration of mucociliary clearance can be induced by other rare pathological conditions which cause the modification of the rheological properties of mucus, making it difficult to eliminate, such as in cystic fibrosis (the viscosity of mucin is 30-60 times greater by altering the transport of $\mathrm{Na}$ and $\mathrm{Cl}$ ions and water), or alteration of kinetics of cils, in primary ciliary dyskinesia or Kartagener syndrome. Cystic fibrosis (CF), although it is cited as the most frequent abnormality monogenic autosomal recessive transmitted in the Caucasian population in Central Europe $(1 / 2,000$ $1 / 2,500$ births), yet it is rarely mentioned in the etiology of chronic rhinosinusitis of the child, if not associating specific and systemic symptoms or superinfection (pseudomonas aeruginosa). Segal et al (8) estimated that CF was diagnosed in $1 / 16$ patients examined for nasal polyposis. In fact, it appears that only $10 \%$ of children with $\mathrm{CF}$ express clinical signs of chronic rhinosinusitis, raising the 
question what happens to the other $90 \%$ of the patients - are they clinically asymptomatic or have adapted to their symptoms by tolerating forms of from mild or moderate rhinosinusitis (9). In particular, 6-67\% of these patients can develop sino-nasal polyposis in chronic evolution of the disease. Out of these cases, $19 \%$ are diagnosed endoscopically until the age of 6 years old and $45 \%$ up to 18 years (10). Chronic polyp expansion gives a particular phenotype, with enlarged base of the nose, hypertelorism, predominance of chronic nasal obstruction, headache and facial pain. Another feature of chronic rhinosinusitis in $\mathrm{CF}$ are mucocel formation by deposition of thick mucus in the sinuses. If they reach a considerable size they can even affect facial area with unilateral swelling or pseudotumoral aspect. Mucocels can be objectified with specific imagistic methods, such as radiography or CT scan. About $12 \%$ of children with CF may develop medial swalling of the sinus wall, as well as secondary mucocel of the maxillary sinus (11). On the other hand, more than $90 \%$ of CF patients presented sinus imaging with opacification, especially in those F 508 homozygote individuals, as well as poor pneumatisation and hypoplasia of the sinus $(12,13)$. Frequently, adolescents with CF dont have frontal sinus pneumatisation, which can indicate the suspicion of disease and atypical forms of CF manifestations, or as CFTR-related disease (chronic rhinosinusitis bronchopulmonary aspergillosis, idiopathic bronchiectasis). In conclusion, CF (atypical form) can be expressed as refractory chronic rhinosinusitis, with normal or slightly elevated iontophoresis values between $40-60 \mathrm{mmol} / \mathrm{l}$, a situation which requires genetic investigation (usually 38 mutations) (14). Raman V et al (15) identified $12 \%$ carriers of a single mutation CF in a study of 58 children with rhinosinusitis. Sinus bacterial superinfection is common in chronic rhinosinusitis. The ideal microbiological diagnosis is performed in an invasive manner, considering the fact that differences between pathogens were reported in the same patients, as well as different colonisation in sinuses of the same patient. Bacterial etiology in these cases is suggestive and sometimes particular to the patient with chronic rhinosinusitis vs FC Pseudomonas sp (65\%), nontypable $\mathrm{H}$. influenzae (50\%), anaerobes (Peptostreptococcus, Bacteroides sp, 25\%), s. Aureus, Burkholderia cepacia, Achromobacter xylosoxydans, sternotrophomonas maltophylia. However, in $33 \%$ of cases of CF associated chronic rhinosinusitis there were reported positive fungal cultures (16). Given these features, the management of CF and chronic rhinosinusitis is com- plex and it aims eliminating chronic obstruction by secretion drainage, airway inflammation control, effective treatment in associated infections, nutritional and immunological support. So the pathogenic treatment with alpha dornase will combine respiratory physiotherapy and antibiotics in microbiologically proven specific situations, especially local therapeutic actions. Dornase alfa (Pulmozyme, rh-DNAse) administered by nebulization improves the rheological properties of mucus by decreasing its viscosity, thereby having an anti-inflammatory effect and improving ciliary clearance, reducing the risk of infection (17). Antibiotic therapy of chronic rhinosinusitis is intensely debated today especially for the inhaled form of administration. Although there are studies that support the beneficial role of certain inhaled antibiotics as tobramycin (Tobi), Colistin, Aztreonam, yet still are not enough evidence to support their effectiveness in chronic rhinosinusitis with polyposis of FC patients (18). Consequently, systemic antibiotic therapy lasting 3-6 weeks remains the recognized treatment in these cases. Among described antibiotics, macrolides and quinolones, as well as IL8 agents mediating anti-inflammatory effect have demonstrated to decrease the size of polyps (19). Immunoprophylaxy vaccines and supporting those with immune deficiency (associated with administration of intravenous immunoglobulin) should be considered in these patients. Additional conservative therapeutic measures have improved the prognostic score. It can be used saline lavage $0.9 \%$ or hypertonic $3 \%$, having an decongestant effect (20), with or without other nasal decongestants (oxymetazoline, xylometazoline, phenylephrine etc.) within one week (depending on risk in prolonged use), but unfortunately without direct effect on the maxillary and ethmoidal sinus. Topical nasal corticosteroid therapy (betamethasone) proved its efficiency by decreasing polyp size perhaps by having anti-inflammatory effect (21), although it remains controversial in systemic (short course) therapy in combination with antibiotics. NSAIDs (ibuprofen) appears effective in patient with compromised lung function, but at higher doses, which induce some risk of side effects (22). Dornase alfa therapy decreases the risk of recurrence after polyp surgery, sustaines anti-edematous effect and thereby decreases the necessary for invasive rhinosinusal procedures (23). Approximately 3\% of patients with $\mathrm{CF}$ and chronic rhinosinusitis associated with polyps require surgical treatment, as $10-20 \%$ of those are non-responders to medical treatment (18). In particular, those are patients whose quality of life is 
deeply disturbed by facial pain or headache, as in severe chronic rhinosinusitis directly correlated with decreased lung function, especially before lung transplantation (24). CF therapeutic perspectives are represented by the use of modulators of CFTR for targeted groups of patients selecting a particular type of mutation, possibly efficient also in chronic rhinosinusitis (Ivacaftor VX-770 for the mutation G551D, Lumacaftor VX-809, AtalurenPTC124), currently approved by the FDA in the US for children older than 6 years (25). Gene therapy using a viral vector (adenovirus) aimes mainly the respiratory epithelium damage, but can also im-

\section{REFERENCES}

1. Criddle M.W., Stinson A., Savliwala M., Coticchia J. Pediatric chronic rhinosinusitis: a restropective review, American Journal of Otolaryngology, 2008; 29(6):372-378.

2. Ahmad R. Sedaghat, Wanda Phipatanakul, Michael J. Cunningham, Prevalence of and Associations with Allergic Rhinitis in Children with Chronic Rhinosinusitis, Boston Children Hospital, American Society of Pediatric Otolaryngology 28 th Spring Meeting, Arlington, april, 2013

3. McClay J.E., Marple B., Kapadia L, et al. Clinical presentation of allergic fungal sinusitis in children. Laryngoscope, 2002, 112(3):565-9

4. Feng C.H., Miller M.D., Simon R.A. The united allergic airway: connections between allergic rhinitis, asthma, and chronic sinusitis, Am J Rhinol Allergy. 2012; 26(3):187-90.

5. EFA Book on Respiratory Allergies-Raise Awareness, Relieve the Burden, edited by Erkka Valovirta, 2011

6. Yang-Gi Min, The Pathophysiology, Diagnosis and Treatment of Allergic Rhinitis, Allergy Asthma Immunol Res. 2010, 2(2): 65-76

7. Bousquet J.L., Schünemann H.J., Samolinski B., et al. World Health Organization Collaborating Center for Asthma and Rhinitis. Allergic Rhinitis and its Impact on Asthma (ARIA): achievements in 10 years and future needs, J. Allergy Clin Immunol. 2012, 130(5):1049-62.

8. Segal N., Gluk O., Puterman M. Nasal polyps in the pediatric population, B-ENT, 2012. 8(4):265-7.

9. Kang S.H., Dalcin Pde T., Piltcher O.B., Migliavacca Rde O. Chronic rhinosinusitis and nasal polyposis in cystic fibrosis: update on diagnosis and treatment, J Bras Pneumol, 2015, 41(1):65-76.

10. Schraven S.P., Wehrmann M., Wagner W., Blumenstock G., Koitschev A. Prevalence and histopathology of chronic polypoid sinusitis in pediatric patients with cystic fibrosis, J Cyst Fibros. 2011, 10(3):181-6.

11. Oomen K.P., April M.M. Sinonasal manifestations in cystic fibrosis, Int J Otolaryngol, 2012;

12. Babinski D., Trawinska-Bartnicka M. Rhinosinusitis in cystic fibrosis: Not a simple story. Intl J Ped Otorhinol, 2008, 72:619-624

13. Woodworth B.A., Ahn C., Flume P.A., Schlosser R.J. The delta F508 mutation in cystic fibrosis and impact on sinus development, $A m$ J Rhinol, 2007; 21(1):122-127

14. Boyle M.P. Nonclassic cystic fibrosis and CFTR - related diseases, Curr Opin Pulm Med 9:498-503, 2003 prove pain and reduce the incidence of ciliary chronic rhinosinusitis (26).

\section{CONCLUSIONS}

The chronic rhinosinusitis clinical heterogenity of expression in relation on the one hand, with immunogenic response and anatomical particularities of the child, and secondly the diversity of etiologic factors (from very frequent-allergy, to least frequent - FC) requires proper diagnosis, early and appropriate treatment, and a multidisciplinary team collaboration (pediatrician, ENT physician, allergist, pulmonologist).

15. Raman V., Clary R., Siegrist K.L., et al. Increased prevalence of mutations in the cystic fibrosis transmembrane conductance regulator in children with chronic rhinosinusitis, Pediatrics, 2002, 109 (1),1-4

16. Johansen H.K., Aanaes K., Pressler T., Nielsen K.G., Fisker J., Skov M., et al. Colonisation and infection of the paranasal sinuses in cystic fibrosis patients is accompanied by a reduced PMN response, $J$ Cyst Fibros, 2012; 11(6):525-531

17. Mainz J.G., Schiller I., Ritschel C., et al. Sinonasal inhalation of dornase alfa in CF: A double-blind placebo-controlled cross-over pilot trial. Auris Nasus Larynx, 2011, 38:220-227

18. Mainz J.G., Koitschev A. Pathogenesis and management of nasal polyposis in cystic fibrosis, Curr Allergy Asthma Rep., 2012; 12(2):163-174

19. Yamada T., Fujieda S., Mori S., Yamamoto H., Saito H. Macrolide treatment decreased the size of nasal polyps and IL-8 levels in nasal lavage, Am J Rhinol, 2000; 14(3):143-148

20. Harvey R., Hannan S.A., Badia L., Scadding G. Nasal saline irrigations for the symptoms of chronic rhinosinusitis, Cochrane Database Syst Rev., 2007; (3)

21. Hadfield P.J., Rowe-Jones J.M., Mackay I.S. A prospective treatment trial of nasal polyps in adults with cystic fibrosis. Rhinology, 2000; 38(2):63-65

22. Lindstrom D.R., Conley S.F., Splaingard M.L., Gershan W.M. Ibuprofen therapy and nasal polyposis in cystic fibrosis patients, J Otolaryngol, 2007; 36(5):309-314.

23. Raynor E.M., Butler A., Guill M., Bent 3rd J.P. Nasally inhaled dornase alfa in the postoperative management of chronic sinusitis due to cystic fibrosis. Arch Otolaryngol Head Neck Surg. 2000; 126(5):581-583.

24. Konstan M.W., Morgan W.J., Butler S.M., et al. Risk factors for rate of decline in forced expiratory volume in one second in children and adolescents with cystic fibrosis. J Pediatr 151, 2007 :134-139

25. Chaaban M.R., Kejner A., Rowe S.M., Woodworth B.A. Cystic fibrosis chronic rhinosinusitis: a comprehensive review. Am J Rhinol Allerg., 2013; 27(5):387-395

26. Wagner J.A., Nepomuceno I.B., Shah N., Messner A.H. et al. Maxillary sinusitis as a surrogate model for CF gene therapy clinical trials in patients with antrostomies. J Gene Med., 1999; 1(1):13-21 